\title{
Optical transport of fluorescent diamond particles inside a tapered capillary
}

\author{
Christophe Pin $^{1}$, Ryohei Otsuka ${ }^{1}$,Hideki Fujiwara ${ }^{1}$, Keiji Sasaki ${ }^{1, *}$ \\ ${ }^{1}$ Research Institute for Electronic Science, Hokkaido University, Kita 20-jo Nishi 10-chome, Kita-ku, Sapporo, Hokkaido, \\ 001-0020, Japan
}

\begin{abstract}
Optical forces provide an efficient way to sort particles and biological materials according to their optical properties. However, both enhanced optical forces and a large interaction volume are needed in order to optically sort a large number of nanoparticles. We investigate the use of a tapered glass capillary as an optofluidic platform for optical manipulation and optical sorting applications. Tapered capillaries with micrometre and sub-micrometre sizes are fabricated. After filling the tapered capillary with a colloidal solution of red fluorescent diamond particles, a green laser light is coupled into the capillary. The tapered capillary acts both as a microfluidic channel and as an optical waveguide, making it possible for the light to interact with the particles inside the sample solution. Using an incident laser power of few tens of milliwatts, we achieve optical transportation of the brightest particles inside the tapered part of the capillary. Particle velocities as high as few tens of micrometres per second are measured.
\end{abstract}

\section{Introduction}

Based on optical forces, optical sorting provides an efficient and contactless method to separate materials in liquid suspension according to their optical properties [18]. Various properties of light such as its wavelength, its polarization, and its spatial distribution, can be used to tailor the optical forces being applied to different particles in solution. As light-matter interactions depend not only on the light properties but also on the optical properties of materials, selective optical trapping or transportation can be achieved and used in order to optically sort nanoparticles with different optical properties. Such an optical sieve is highly needed for sorting a large variety of nanomaterials. However, efficient optical manipulation of a large number of nanoparticles remains a challenging task since it would require both an intense laser light to cover a large interaction volume, and near-field enhanced optical forces that drastically reduce the size of the interaction volume [4,9-15].

In order to overcome this issue, we focus here on the fabrication and the use of tapered glass capillaries for optofluidic applications such as nanoparticle optical manipulation. First, we present numerical simulation results in order to evaluate the optical properties of glass micro- and nanocappillaries. In a second part, we describe how micrometre and sub-micrometre size glass capillaries can be fabricated. Eventually, we report on the optical transport of fluorescent diamond particles inside a tapered capillary.

\section{Results and discussion}

\subsection{Micro- and nanocapillary waveguides}

We consider here fused-silica glass micro- and nanocapillaries filled with water solution and surrounded by air. The refractive index of both capillary materials $\left(\mathrm{n}_{\text {glass }}\right.$ $\left.=1.46, \mathrm{n}_{\text {water }}=1.33\right)$ being higher than the refractive index of air $\left(\mathrm{n}_{\mathrm{air}}=1.0\right)$, the tapered capillary acts as an optical waveguide with a partially liquid part. We numerically investigate the optical guiding properties of micro- and nanocappillaries in order to evaluate the overlap between the guided light and the liquid channel at different wavelengths and for different capillary sizes. Figure 1 shows the electric field distributions of the fundamental guided mode that can be excited in waterfilled capillaries with different diameters at $1064 \mathrm{~nm}$ and $532 \mathrm{~nm}$ wavelengths.

Although reaching the single-mode regime requires tapered capillaries to have sub-micrometre dimensions, it is found that such nanocappillaries can offer a large overlap between the guided light and le liquid solution. Larger multimode capillaries may still provide an optofluidic platform for studying light-matter interactions as long as a non-negligible part of the guided light propagates in the evanescent field near the glass-water interface. In such a case, the light propagation in the tapered capillary is expected to depend on the light coupling condition at the entrance of the capillary, as well as on the taper geometry.

Corresponding author: sasaki@es.hokudai.ac.jp 

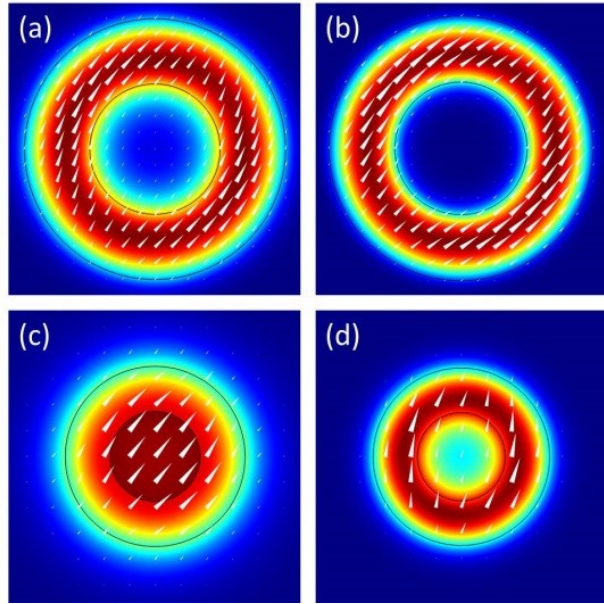

Fig. 1. Cross-sections of the electric field distribution of the fundamental guided mode (a),(b) in a $2 \mu \mathrm{m}$ large capillary and (c),(d) in a $700 \mathrm{~nm}$ large capillary at (a),(c) $1064 \mathrm{~nm}$ and (b),(d) $532 \mathrm{~nm}$ wavelengths.

\subsection{Fabrication of liquid-filled tapered capillaries}

We investigated the fabrication of tapered glass capillaries (as shown in Figure 2) using a standard heating and pulling process originally developed in order to fabricate tapered optical fibres. Figure 3 shows the pulling-time dependence of the waist outer diameter of the fabricated tapered capillaries. Tapered capillaries with sub-micrometre diameters down to $200 \mathrm{~nm}$ were successfully fabricated after optimization of the pulling time and speed.

A home-made system was developed in order to inject a liquid sample solution inside a tapered capillary. For the experimental demonstration of particle optical transport inside a tapered capillary, fluorescent diamond particles were chosen for their high refractive index value $(2.44$ at $532 \mathrm{~nm})$ and their bright and robust fluorescence due to the presence of NV- centers (no photobleaching or blinking effects) [16]. We used a water solution containing red fluorescent diamond particles with an average size of $100 \mathrm{~nm}$ that was purchased from FND Biotech.

After injecting the liquid solution inside the capillary, both ends of the capillary were cleaved, and green laser light (at $532 \mathrm{~nm}$ wavelength) was coupled to one extremity of the capillary. We used a cleaved optical fibre coated with immersion oil to improve the coupling efficiency. Observation and recording of the particles' motion inside the capillary was done using a standard fluorescence microscope.
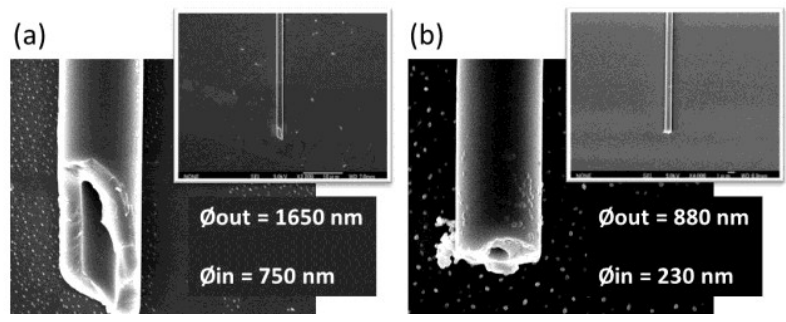

Fig. 2. Scanning electron microscopy images of two broken tapered glass capillaries of different sizes.

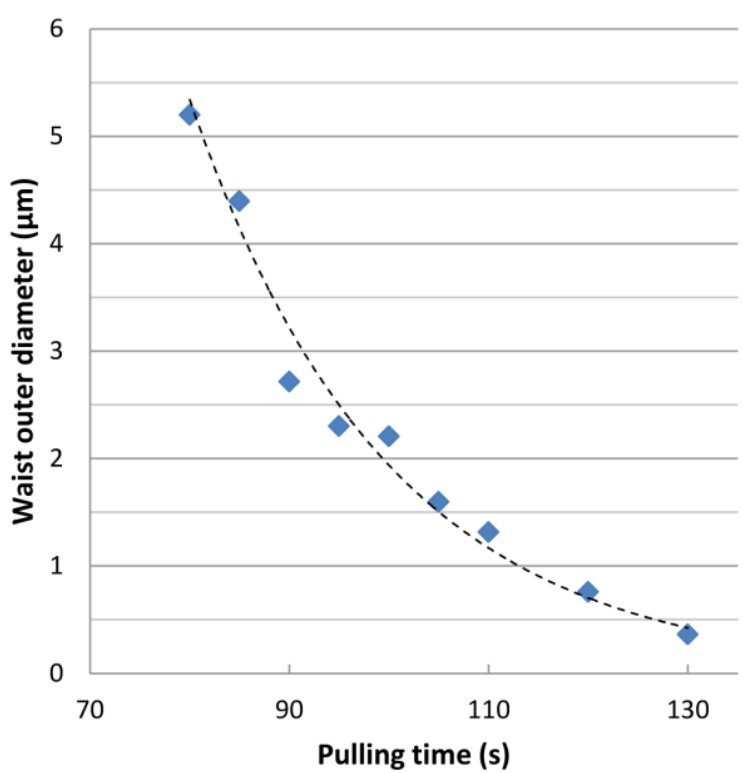

Fig. 3. Waist outer diameter of the fabricated tapered capillaries as a function of the pulling time (pulling speed: 340 $\mu \mathrm{m} / \mathrm{s})$.

\subsection{Optical transport of fluorescent diamonds}

During the experiment, a transmission efficiency reaching about $20 \%$ was measured. This value takes into account both the fibre-to-capillary coupling losses and the radiative losses occurring in the tapered part of the capillary. Using a tapered capillary of $6.7 \mu \mathrm{m}$ outer diameter, the brightest diamond particles were successfully propelled inside the capillary when the laser was switched on. We used an input laser power of few tens of milliwatts. Similar results were obtained using a tapered capillary with a $2.2 \mu \mathrm{m}$ outer diameter. The recorded particles' motion was then analysed, and particle velocities reaching few tens of micrometres per second were measured. We were able to control the particle velocity by varying the input laser power.

\section{Conclusion}

In this work, we investigated the fabrication and use of tapered glass capillaries for nanoparticle optical transport and sorting applications. We first studied the optical properties of tapered capillaries using a numerical model. Then we fabricated tapered glass capillaries filled with a colloidal solution of red fluorescent diamond particles, and we performed optical transport experiments using a green laser light coupled into the capillary waveguide. The results presented here are expected to pave the way for further investigation on the use of tapered glass capillaries as an optofluidic platform for optical sorting and other applications.

We acknowledge financial support from JSPS KAKENHI (Grant Numbers JP16H06506, JP18H05205, and JP18H03882). 


\section{References}

1. T. Imasaka, Y. Kawabata, T. Kaneta, Y. Ishidzu, Anal. Chem. 67, 1763 (1995)

2. S.J. Hart, A.V. Terray, Appl. Phys. Lett. 83, 5316 (2003)

3. M.P. MacDonald, G.C. Spalding, K. Dholakia, Nature 426, 421 (2003)

4. M. Ploschner, T. Cizmar, M. Mazilu, A. Di Falco, K. Dholakia, Nano Lett. 12, 1923 (2012)

5. G. Tkachenko, E. Brasselet, Nat. Commun. 5, 3577 (2014)

6. S.E.S. Spesyvtseva, S. Shoji, S. Kawata, Phys. Rev. Appl. 3, 044003 (2015)

7. F. Nan, Z. Yan, Nano Lett. 18, 7400 (2018)

8. Y. Shi, S. Xiong, L.K. Chin, J. Zhang, W. Ser, J. $\mathrm{Wu}$, T. Chen, Z. Yang, Y. Hao, B. Liedberg, P.H. Yap, D.P. Tsai, C.-W. Qiu, A.Q. Liu, Sci. Adv. 4, eaao0773 (2018)

9. A.H. Yang, S.D. Moore, B.S. Schmidt, M. Klug, M. Lipson, D. Erickson, Nature 457, 71 (2009)

10. S.E. Skelton, M. Sergides, R. Patel, E. Karczewska, O.M. Marago, P.H. Jones, J. Quant. Spectrosc. Radiat. Transf. 113, 2512 (2012)

11. A. Cuche, A. Canaguier-Durand, E. Devaux, J.A. Hutchison, C. Genet, T.W. Ebbesen, Nano Lett. 13, 4230 (2013)

12. P. Schein, D. O'Dell, D. Erickson, ACS Photon. 4, 106 (2017)

13. M. Soltani, J. Lin, R.A. Forties, J.T. Inman, S.N. Saraf, R.M. Fulbright, M. Lipson, M.D. Wang, Nature Nanotech. 9, 448 (2014)

14. J.E. Baker, R.P. Badman, M.D. Wang, WIREs Nanomed. Nanobiotechnol. 10, e1477 (2018)

15. C. Pin, J.-B. Jager, M. Tardif, E. Picard, E. Hadji, F. de Fornel, B. Cluzel, Lab. Chip 18, 1750 (2018)

16. W.W.W. Hsiao, Y.Y. Hui, P.C. Tsai, H.C. Chang, Acc. Chem. Res. 49, 400 (2016) 